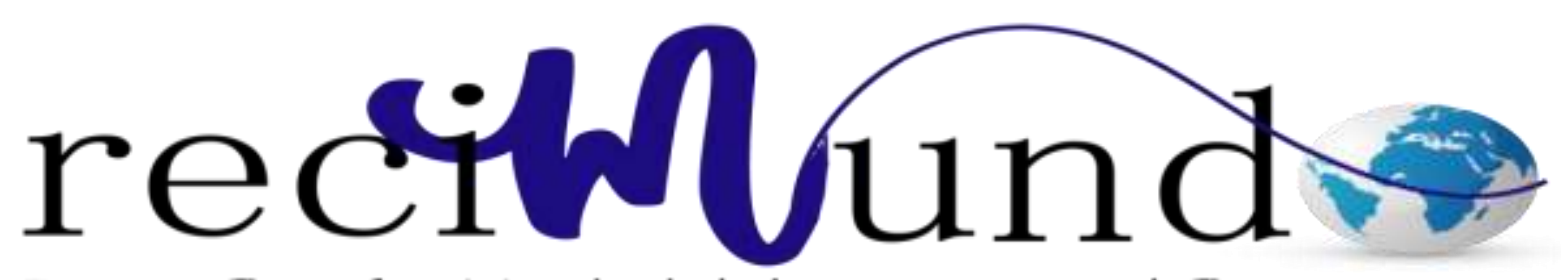

Revista Cientifica Mundo de la Investigación y el Conocimiento

Mariela del Pilar Bedoya Paucar a; Laila Stefanía Lucio Escudero b; Nancy Renee Cando Yaguar $^{\mathrm{c}}$

Gestión del barrio saludable y la organización socio comunitaria en el sector Los Tanques, parroquia Veintimilla, Ciudad Guaranda, Provincia Bolívar, periodo 2013

Healthy neighborhood management and community-based organization in the Los Tanques sector, Veintimilla parish, Guaranda City, Bolivar Province, 2013 period

Revista Científica Mundo de la Investigación y el Conocimiento. Vol. 3 núm.3, septiembre, ISSN: 2588-073X, 2019, pp. 545-556

DOI: $10.26820 /$ recimundo/3.(3).septiembre.2019.545-556

URL: http://recimundo.com/index.php/es/article/view/547

Código UNESCO: 3205 Medicina Interna

Tipo de Investigación: Artículo de Revisión

Editorial Saberes del Conocimiento

Recibido: 15/05/2019

Aceptado: 23/06/2019

Publicado: 30/09/2019

Correspondencia: mariela.bedoyap@ug.edu.ec

a. Magister en Enfermería; Licenciada en Enfermería; Universidad de Guayaquil; Guayaquil, Ecuador; mariela.bedoyap@ug.edu.ec

b. Especialista en Atención Primaria de la Salud; Licenciada en Ciencias de la Enfermería; Universidad de Guayaquil; Guayaquil, Ecuador; 1aila.lucioe@ug.edu.ec

c. Especialista en Enfermería Psiquiátrica y Salud Mental; Licenciada en Enfermería; Universidad de Guayaquil; Guayaquil, Ecuador; nancy.cando@ug.edu.ec 
Gestión del barrio saludable y la organización socio comunitaria en el sector Los Tanques, parroquia Veintimilla, Ciudad Guaranda, Provincia Bolívar, periodo 2013

Vol. 3, núm. 3., (2019)

Mariela del Pilar Bedoya Paucar; Laila Stefanía Lucio Escudero; Nancy Renee Cando Yaguar

\section{RESUMEN}

Las ciudades son lugares de concentración de actividad económica, creatividad y talento. Son sistemas en los que la densidad, la interacción y la diversidad generan economías de escala y se impulsa la productividad, motores de la economía que crean espacios de conectividad, creatividad e innovación y centros de servicios. El Banco Interamericano de Desarrollo propone 8 aspectos claves dentro de estos programas: equidad, espacios públicos de calidad, conectividad, seguridad, movilidad, resiliencia, conocimiento y gobernanza. El programa de Barrio Saludable por medio de sus técnicos y el voluntariado social, participa en la promoción de ideas y organización de las mingas para incentivar la limpieza en las viviendas y en los barrios. La universidad, con sus escuelas de Salud y Enfermería, también participa en la atención y capacitación a los moradores de los sectores populares. El objetivo es tratar de identificar problemas, riesgos y también elementos beneficiosos en un espacio específico de la ciudad (potencialidades). Esto se consultará con dirigentes barriales, trabajadores del mercado, autoridades de colegios, representantes de los comercios y personal del resto de secretarias del municipio, la iniciativa tiene el auspicio de la Organización Panamericana de la Salud (OPS) para su ejecución. Se evidencia varias formas de asociatividad, entre las más destacadas tenemos: las mingas, las alianzas estratégicas, redes de servicio y presta manos. Los líderes comunitarios son personas que pertenecen a una comunidad, saben compartir y están dispuestos a colaborar con los demás. Estas características producen en el resto de su comunidad confianza y seguimiento de sus indicaciones. Esta participación canalizada a través de una acción de índole comunitaria debe conducir a la auto responsabilidad para solventar los problemas ya sean individuales, grupales o comunales. La metodología usada es descriptiva, con un enfoque documental, es decir, revisar fuentes disponibles en la red, como google académico, con contenido oportuno y relevante desde el punto de vista científico y actualizado que enriquezca el análisis del tema planteado en este artículo.

Palabras claves: Comunidad; Búsqueda de soluciones; Calidad de vida; Prevención; Seguridad; Sanidad; Salud. 


\title{
Gestión del barrio saludable y la organización socio comunitaria en el sector Los Tanques, parroquia Veintimilla, Ciudad Guaranda, Provincia Bolívar, periodo 2013
}

Vol. 3, núm. 3., (2019)

Mariela del Pilar Bedoya Paucar; Laila Stefanía Lucio Escudero; Nancy Renee Cando Yaguar

\begin{abstract}
Cities are places of concentration of economic activity, creativity and talent. They are systems in which density, interaction and diversity generate economies of scale and boosts productivity, engines of the economy that create spaces for connectivity, creativity and innovation and service centers. The Inter-American Development Bank proposes 8 key aspects within these programs: equity, quality public spaces, connectivity, security, mobility, resilience, knowledge and governance. The Healthy Neighborhood program through its technicians and social volunteering, participates in the promotion of ideas and organization of the mingas to encourage cleanliness in homes and neighborhoods. The university, with its schools of Health and Nursing, also participates in the care and training of the inhabitants of the popular sectors. The objective is to try to identify problems, risks and also beneficial elements in a specific space of the city (potentialities). This will be consulted with neighborhood leaders, market workers, school authorities, representatives of businesses and staff of the rest of the secretaries of the municipality, the initiative is sponsored by the Pan American Health Organization (PAHO) for its execution. There are several forms of associativity, among the most prominent we have: mingas, strategic alliances, service networks and lend hands. Community leaders are people who belong to a community, know how to share and are willing to collaborate with others. These characteristics produce confidence and follow-up of their indications in the rest of their community. This participation channeled through an action of a community nature should lead to self-responsibility to solve problems, whether individual, group or communal. The methodology used is descriptive, with a documentary approach that is, reviewing sources available on the web, such as google scholar, with timely and relevant content from the scientific and updated point of view that enriches the analysis of the topic raised in this article.
\end{abstract}

Key words: Community; Search for solutions; Quality of life; Prevention; Security; Health; Health. 


\section{Gestión del barrio saludable y la organización socio comunitaria en el sector Los Tanques, parroquia Veintimilla, Ciudad Guaranda, Provincia Bolívar, periodo 2013}

Vol. 3, núm. 3., (2019)

Mariela del Pilar Bedoya Paucar; Laila Stefanía Lucio Escudero; Nancy Renee Cando Yaguar

\section{Introducción.}

En Ecuador existe una gran cantidad de programas de apoyo para las asociaciones indistintamente de su naturaleza o actividad que desarrollen entre estos se pueden destacar el Ministerio del Ambiente con el Programa Nacional para la Gestión Integral de Desechos Sólidos en Ecuador (PNGIDS), en donde se basan las estrategias en las posibles asociaciones, tomando como referencia la inversión privada, trabajo, y gestión comunitaria que serán esenciales a la hora de desarrollar el modelo por parte de los gobiernos locales, de conformidad con el Plan Nacional del Buen Vivir (Borjas, Julio 2007).

Son varias las entidades que se encargan de regular las asociaciones entre ellos están las asociaciones, organizaciones civiles que están registradas, acreditadas y controladas por el Sistema Unificado de Información de las organizaciones sociales (SUIOS), cuya rectoría ejercerá la Secretaría Nacional de Gestión Política. Este se encuentra descrito en el decreto presidencial número 16, publicado el 4 de junio de 2013. Para generar e implementar políticas y programas que aborden la equidad en salud de manera holística y con la participación de los residentes, la ciudad está produciendo, recopilando y procesando información sobre la salud para establecer un diálogo con los residentes y escuchar sus prioridades respecto al estado de la salud en diferentes barrios, a través de la coordinación intersectorial.

Las organizaciones socio comunitarias representa una solución descentralizada para abordar los distintos problemas existentes en las ciudades. Se busca aprovechar el talento humano y las fortalezas de la zona junto con la obtención de información de primera mano: sus protagonistas, quienes deberán priorizar múltiples necesidades con fines cada vez más limitados como se explicará a continuación. Es más conveniente acercarse a la comunidad partiendo del rol de representantes comunales que harán de puente entre la comunidad y los diferentes entes reguladores municipales, regionales o nacionales.

\section{Metodología.}

Esta investigación está dirigida al estudio de la "Gestión del Barrio Saludable y la Organización Socio Comunitaria en el sector Los Tanques, parroquia Veintimilla, Ciudad Guaranda, Provincia Bolívar, periodo 2013”. Para realizarlo se usó una metodología tipo descriptiva, con un enfoque documental, es decir, revisar fuentes disponibles en la red, como google académico, con contenido oportuno y relevante desde el punto de vista científico para dar respuesta a lo tratado en el presente artículo y que sirvan de inspiración para realizar otros proyectos. Las mismas pueden ser consultadas al final, en la bibliografía.

\section{Resultados.}

En 2013, la Asamblea General de las Naciones Unidas declaró el 31 de octubre como el día Mundial de las Ciudades, bajo el lema de Mejor ciudad, mejor calidad de vida. La acelerada urbanización en América Latina, comparada con la de otras latitudes, y de la falta de capacidad de 


\section{Gestión del barrio saludable y la organización socio comunitaria en el sector Los Tanques, parroquia Veintimilla, Ciudad Guaranda, Provincia Bolívar,}

periodo 2013

Vol. 3, núm. 3., (2019)

Mariela del Pilar Bedoya Paucar; Laila Stefanía Lucio Escudero; Nancy Renee Cando Yaguar

los gobiernos locales y nacionales para poder dar respuestas oportunas a este proceso, se evidencia en los siguientes datos: 8 de cada 10 latinoamericanos habitan en ciudades y un $70 \%$ de la población de escasos recursos vive en zonas urbanas. El $80 \%$ del producto interno bruto de los países se genera en estos espacios metropolitanos (Alder, 2013).

Las ciudades son lugares de concentración de actividad económica, creatividad y talento. Son sistemas en los que la densidad, la interacción y la diversidad generan economías de escala y se impulsa la productividad, motores de la economía que impulsan espacios de conectividad, creatividad e innovación y centros de servicios. El Banco Interamericano de Desarrollo (BID) propone evaluar 8 aspectos claves para mejorar las ciudades:

\begin{tabular}{|l|}
\hline 1. Equidad: minimizar las brechas que ocasionen desigualdad entre la población. \\
\hline 2. Espacios públicos de calidad: acondicionar estos espacios como lugares de \\
encuentro.
\end{tabular}

Fuente: (Alder, 2013)

\section{Programa Barrio Saludable}

Es un programa que involucra a las instituciones Malaria, Gobierno Provincial Autónomo, Universidades, Ministerio de Inclusión Económica y Social, Aguas y Servicios, Dirección de Educación, Juntas Parroquiales, Empresa de Aseo y Gobernación. La iniciativa incluye a las instituciones que pueden actuar en la prevención de enfermedades y en la atención durante situaciones de emergencia (El Telégrafo, 2013).

Dirigiendo el proyecto estará la Dirección Provincial de Salud, la Secretaría de Desarrollo Social y el Patronato de Acción Social, entre otros, coordinando el ejercicio. Funcionarios del Gobierno Provincial acudirán a los barrios para la organización de los espacios saludables como medio para capacitar a los habitantes. El Municipio colabora con técnicos y promotores para la operación de la propuesta. Por medio de sus técnicos y el voluntariado social, participa en la promoción de la idea y en la organización de las mingas para incentivar la limpieza en las viviendas y en los barrios. La universidad, con sus escuelas de Salud y Enfermería, también participa en la atención y capacitación a los moradores de los sectores populares. Malaria fumiga viviendas y barrios. Los médicos de la Dirección de Salud y el Patronato se encargarán de atender a pacientes. 


\section{Gestión del barrio saludable y la organización socio comunitaria en el sector Los Tanques, parroquia Veintimilla, Ciudad Guaranda, Provincia Bolívar, periodo 2013}

Vol. 3, núm. 3., (2019)

Mariela del Pilar Bedoya Paucar; Laila Stefanía Lucio Escudero; Nancy Renee Cando Yaguar

Para el último trimestre del año 2016, se empezó a trabajar en tres barrios de la ciudad (en el centro, sur y norte de la ciudad de Quito) para ir promoviendo estos temas y tratar la salud no como un problema de enfermedad o curación solamente, sino también de cómo contribuir a la salud en el espacio urbano (Coello, 2016).

El objetivo es tratar de identificar problemas, riesgos y también elementos beneficiosos en un espacio específico de la ciudad (potencialidades). Esto se consultará con dirigentes barriales, trabajadores del mercado, autoridades de colegios, representantes de los comercios y personal del resto de secretarias del municipio, la iniciativa tiene el auspicio de la Organización Panamericana de la Salud (OPS) para su ejecución y que se seguirán "algunos lineamientos de la nueva Agenda Urbana que precisamente se va a firmar durante el evento Habitad III en Quito, ese año.

Durante el año 2017 la Secretaria de Salud ha informado que se ha dado inicio a la implementación del proyecto Barrio Saludable y se han compartido experiencias de las administraciones zonales en "la construcción de una hoja de ruta. El rol protagónico que cumple el comité local de salud" de su ciudad, conformado por 3 universidades de Gante, 3 organizaciones de salud escolar, el centro de bienestar social, la organización de promoción de salud, la Organización Niño y Familia, una organización del gobierno flamenco con consultorios para niños en toda la ciudad y la organización representando a los pacientes.

Según comunicado oficial, los actores institucionales municipales deberán tener la capacidad de identificar y reducir inequidades en salud e incidir efectivamente sobre los determinantes de la salud, mediante la identificación de riesgos y la construcción de una estrategia de respuesta de manera participativa, en materia de equidad en salud en barrios de la ciudad. La institución también ha considerado que la "participación ciudadana, el levantamiento y análisis de datos de salud, el trabajo articulado intersectorial en coordinación con el sector privado y el desarrollo e implementación de planes de acción participativos serán los componentes claves del programa (Ruales, 2017).

\section{Organización Socio Comunitaria}

El término asociatividad es un concepto que se ha ido difundiendo y posicionando en las comunidades, empresarios, organismos nacionales e internacionales bajo principios de cooperación, solidaridad, trabajo en equipo, en la búsqueda de alternativas de solución a los problemas que aqueja a cada uno de ellos, creando ventajas competitivas sostenibles en el tiempo. La Asociatividad de las organizaciones sociales en el Ecuador enuncia cooperación, unión, reciprocidad entre individuos y organizaciones que se juntan voluntariamente con el fin de satisfacer sus necesidades, lograr un solo propósito, bajo la participación y el esfuerzo colectivo.

En el Ecuador existe una gran cantidad de programas de apoyo para las Asociaciones indistintamente de su naturaleza o actividad que desarrollen entre estos se pueden destacar el Ministerio del Ambiente con el Programa Nacional para la Gestión Integral de Desechos Sólidos en Ecuador (PNGIDS), en donde se basan las estrategias en las posibles asociaciones, tomando

Revista Científica Mundo de la Investigación y el Conocimiento. 3 (3). pp. 545-556 


\section{Gestión del barrio saludable y la organización socio comunitaria en el sector Los Tanques, parroquia Veintimilla, Ciudad Guaranda, Provincia Bolívar, periodo 2013}

Vol. 3, núm. 3., (2019)

Mariela del Pilar Bedoya Paucar; Laila Stefanía Lucio Escudero; Nancy Renee Cando Yaguar

como referencia la inversión privada, trabajo, y gestión comunitaria que serán esenciales a la hora de desarrollar el modelo por parte de los gobiernos locales, de conformidad con el Plan Nacional del Buen Vivir, la Corporación Financiera Nacional (CFN), BanEcuador, Instituto de Economía Popular y Solidaria (IEPS) ofrecen programas de fomento productivo para incentivar la producción y el desarrollo socio económico de sus comunidades, apoyando a los productores, asociaciones y sectores de menor desarrollo, a través de la cooperación interinstitucional entre la Corporación y los Promotores de Programas de Desarrollo (Borjas, Julio 2007). Se evidencia varias formas de asociatividad, entre las más destacadas tenemos:

- Las mingas: basada en el trabajo comunitario o colectivo, con fines de utilidad social, permite hacer el trabajo más rápido y mejor, gracias al llamado de los líderes, la población se moviliza, organiza de manera expedita, se celebra el trabajo y los logros.

- Alianzas estratégicas: son relaciones horizontales, entre empresas que compiten en el mercado, pero que se unen y cooperan en ciertas actividades complementarias o en algunas que no tienen experticia, como pueden ser investigación y desarrollo, compras, comercialización, etc.

- Redes de servicios: son grupos de personas con especialización y conocimientos específicos que proporcionan servicios puntuales, de acuerdo al campo de ocupación, necesidad. Economías de escala: son grupos de comunidades que necesitan adquirir productos o servicios similares y se reúnen con el objeto de aumentar el poder de negociación frente a los proveedores.

- Presta manos: son formas de cooperación comunitaria, que se fundamenta en la devolución del trabajo reciproco; si una persona o comunidad requiere el servicio o trabajo para construir, cultivar o realizar alguna actividad en beneficio de la organización o personas, solicita el apoyo del grupo de personas o comunidades con el compromiso de devolver la misma cantidad de trabajo, tiempo y materiales en actividades que la comunidad o persona aportante requiera posteriormente.

Los líderes comunitarios son aquellas personas que pertenecen a una comunidad, saben compartir lo que tienen y están dispuestos a colaborar con los demás. Estas características producen en el resto de su comunidad confianza y seguimiento de sus indicaciones. La influencia que un líder puede tener en el resto de su comunidad le permite tomar un rol fundamental en la participación y desarrollo de su propia comunidad. Esta participación canalizada a través de una acción de índole comunitaria debe conducir a la auto responsabilidad para solventar los problemas ya sean individuales, grupales o comunales (Martí, 2008)

Conseguir una participación racional de los líderes comunitarios es el punto clave en la movilización de la comunidad, es decir se convierte así el líder, en el centro y motor para desatar el proceso participativo de la comunidad. Es conveniente que a los líderes se les ofrezcan los medios y herramientas necesarios para que intervengan de una manera efectiva en la consolidación de cualquier proyecto de apoyo a la salud comunitaria (Navas, 2013).

Revista Científica Mundo de la Investigación y el Conocimiento. 3 (3). pp. 545-556 


\section{Gestión del barrio saludable y la organización socio comunitaria en el sector Los Tanques, parroquia Veintimilla, Ciudad Guaranda, Provincia Bolívar, periodo 2013}

Vol. 3, núm. 3., (2019)

Mariela del Pilar Bedoya Paucar; Laila Stefanía Lucio Escudero; Nancy Renee Cando Yaguar

\section{Funciones generales:}

- Dinamizadores de la comunidad para lograr un desarrollo y mejorar la calidad de vida de la comunidad.

- Deben representar a la comunidad y ser los interlocutores ante organismos, autoridades y otras instituciones locales, departamentales y nacionales. Serán los encargados de redactar y expresar las necesidades de la comunidad a las que pertenecen ante cualquier organismo o autoridad.

- En colaboración con el resto de la comunidad, deben realizar el diagnóstico de las necesidades de su comunidad para hacer frente a los problemas y/o eventos de emergencia que pudieran plantearse.

- Deben formar parte del comité para la administración y gestión de botiquines comunitarios.

- Deben ser los responsables de programar, organizar, dirigir, administrar, gestionar y evaluar un proyecto comunitario.

- Tienen que velar por la situación medio ambiental de la comunidad y realizar una valoración de la misma periódicamente.

El éxito de esta estructura dentro de la comunidad Caspigasí ubicada en la parroquia de Calacalí, provincia de Pichincha, en las cercanías de la línea equinoccial, les permitió solventar parte de la problemática agraria, minera y ambiental, el desabastecimiento de agua, la contaminación que afectaba su calidad de vida, su experiencia fue: para enfrentar esta situación en una perspectiva de mediano plazo surge la necesidad de levantar nuestro Plan de Ordenamiento Territorial en el año 1985 con el fin de zonificar la tierra y decidir dónde debería ir ubicada la población, donde la conservación para garantizarnos el agua, dónde se realizarían las labores de pastoreo, las plantaciones de hierbas medicinales. Se evaluó que sería de su comunidad luego de la minería, surgió un proyecto turístico comunitario aquí en la mitad del mundo (Castro, 2013).

Para la elaboración del Plan se revisó documentación histórica, se desarrolló un censo comunitario de población y de actividades productivas familiares, se obtuvo una fotografía aérea de la comunidad y se llevaron a cabo recorridos por los distintos sectores de la comunidad. Luego de un complejo proceso de negociaciones al interior de la comunidad, en torno a la necesidad de una zonificación, delimitación y uso de suelos se concluyó exitosamente con un producto final que es el Plan de Desarrollo y Ordenamiento Territorial, (sin recibir ningún tipo de apoyo externo) logrando un resultado auto gestionado en su totalidad. Con la propuesta aprobada por la Asamblea General de la comunidad en el año 2001, tiempo después este plan sería un referente a nivel zonal y provincial.

El tratamiento de las enfermedades y la atención han sido el centro neurálgico de la política de salud. Esta apuesta por la curación no toma en consideración que la mayor parte de afectaciones de la salud son atribuibles al medio socio-económico, por este motivo, las intervenciones deben redirigirse hacia las causas de las causas. Existe un gradiente social de la salud, conforme se desciende por la escala social, aumentan los problemas de salud. Esto responde a una distribución desigual del poder, bienes y servicios. La experiencia de desigualdad se incorpora en las personas

Revista Científica Mundo de la Investigación y el Conocimiento. 3 (3). pp. 545-556 


\section{Gestión del barrio saludable y la organización socio comunitaria en el sector Los Tanques, parroquia Veintimilla, Ciudad Guaranda, Provincia Bolívar, periodo 2013}

Vol. 3, núm. 3., (2019)

Mariela del Pilar Bedoya Paucar; Laila Stefanía Lucio Escudero; Nancy Renee Cando Yaguar

desde la cuna hasta la tumba y se refleja en su salud. Ello no es natural, es síntoma de un sistema estructural que valora unos seres humanos más que a otros. Cerrar estas brechas de inequidad es una tarea impostergable del gobierno central pero también de los gobiernos locales conforme a sus competencias. Este planteamiento se resume en el gráfico $\mathrm{N}^{\circ} 1$ mostrado a continuación.

\section{Gráfico $\mathbf{N}^{\circ} 1$ Modelo Dhalgren yWhitewead}

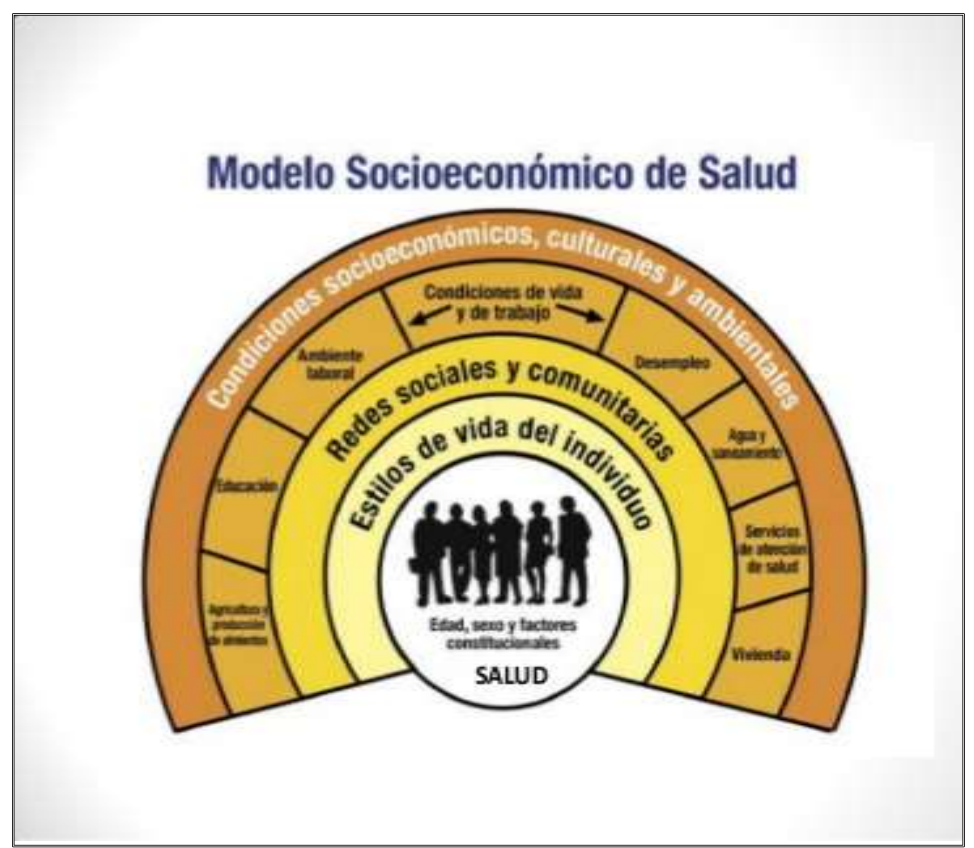

Fuente: (Ministerio de Salud Pública, 2016)

\section{Veintimilla Ciudad Guaranda su problemática}

Para el año 2013 el agua llega durante la noche a ese sector, mientras que en horas de la mañana varias viviendas no cuentan con este servicio. Al visitar cuatro viviendas del sector en dos de ellas se constató la falta total de agua, sin embargo, en los dos restantes al momento de abrir la llave la caída de este líquido vital fue mínima. Sus habitantes debían ir a traer agua del centro de Pujilí. Lo que le representaba un gasto extra para pagar una carrera (La Hora, 2013).

En los tanques de color blanco se observó que el agua muestra un color amarillento, esta es recolectada en horas de la noche. El problema de falta de agua no es reciente, por lo que las personas se han acostumbrado a vivir de esta manera, aunque el comité pro mejoras de la ciudadela ha realizado varios pedidos para el arreglo en el lugar.

El fuerte invierno afecta a las provincias de Bolívar y Cotopaxi durante el año 2017. Vías dañadas, ríos desbordados y casas afectadas dejaron las precipitaciones registradas en los últimos días en estas jurisdicciones de la Sierra centro del Ecuador. Los técnicos del Consejo Provincial y 


\section{Gestión del barrio saludable y la organización socio comunitaria en el sector Los Tanques, parroquia Veintimilla, Ciudad Guaranda, Provincia Bolívar, periodo 2013}

Vol. 3, núm. 3., (2019)

Mariela del Pilar Bedoya Paucar; Laila Stefanía Lucio Escudero; Nancy Renee Cando Yaguar

del Municipio, respectivamente trabajan en la rehabilitación de las carreteras. En Bolívar, la vía Guaranda-Cuatro Esquinas-Salinas fue abierta al tránsito vehicular. Un paso provisional, construido por el personal y la maquinaria del Consejo Provincial, permite la circulación de vehículos livianos.

Con el fuerte invierno que afronta la provincia en esa fecha, se socavó y hundió la calzada. Según el Prefecto, en el recorrido por la vía de 20 kilómetros, se detectó que hay deslaves y problemas de drenaje. Los trabajos realizados están colaborando para que la población no quede asilada y puedan sacar sus productos, viajar por trabajo o negocios. Aclaró que por el momento la circulación será para carros livianos y posteriormente pasarán los más pesados. La carretera a Santa Rosa de Agua Clara está cerrada al tránsito vehicular, un kilómetro de la plataforma se hundió. Otra de los caminos afectados es Chimbo-La Magdalena-El Torneado-Guaranda que está bloqueada (Moreta, 2017).

El temblor de 6.5 grados que ocurrió el 6 de septiembre de 2018 tuvo su epicentro en Cumandá (Chimborazo) y provocó graves daños en Bolívar. Se declaró en emergencia a los cantones de Chillanes y Chimbo. En el COE, Gestión de Riesgos solicitó que se realice una inspección de las fallas geológicas de los cantones más afectados, para tomar acciones preventivas. Otro aspecto a considerar es la temporada de invierno y una crecida del afluente provocaría graves daños a los cultivos y la población.

Docentes y estudiantes de la Universidad Estatal de Bolívar y de la Universidad Técnica de Ambato (UTA) se suman a la tarea del levantamiento de información de los lugares más perjudicados. Algunas casas están inservibles y no pueden vivir por riesgo a que se derrumben en cualquier instante. Otro de los problemas identificados es la situación de pobreza extrema en la que viven las familias, otro de los problemas que identificaron las autoridades es que los terrenos donde se encuentran aquellas casas que deben ser demolidas no se encuentran legalizados (Maisanche, 2018).

\section{Conclusiones.}

Los programas Gestión del Barrio Saludable y la Organización Socio Comunitaria son programas positivos que buscan mejorar la calidad de vida de la población mediante la descentralización. Se busca aprovechar los recursos limitados y direccionarlos a atender aquellas necesidades prioritarias de las comunidades, no todas son iguales ni tienen las mismas problemáticas.

También ofrece otra gran ventaja: facilita la comunicación entre el ente Ministerial u otro Organismo especializado y esa comunidad aprovechando la figura del líder comunitario de mediador, quien debe ser alguien respetable y valorado por su comunidad, al vivir allí padece junto con su entorno pudiendo expresar con precisión esa realidad. Estas personas requerirán de estudio y formación que están a cargo de centros de aprendizaje a nivel técnico y universitario.

Revista Científica Mundo de la Investigación y el Conocimiento. 3 (3). pp. 545-556 


\section{Gestión del barrio saludable y la organización socio comunitaria en el sector Los Tanques, parroquia Veintimilla, Ciudad Guaranda, Provincia Bolívar, periodo 2013}

Vol. 3, núm. 3., (2019)

Mariela del Pilar Bedoya Paucar; Laila Stefanía Lucio Escudero; Nancy Renee Cando Yaguar

El tratamiento de las enfermedades y la atención han sido el centro neurálgico de la política de salud. Esta apuesta por la curación no toma en consideración que la mayor parte de afectaciones de la salud son atribuibles al medio socio-económico, por este motivo, las intervenciones deben redirigirse hacia las causas de las causas. Existe un gradiente social de la salud, conforme se desciende por la escala social, aumentan los problemas de salud. Esto responde a una distribución desigual del poder, bienes y servicios. La experiencia de desigualdad es síntoma de un sistema estructural que valora unos seres humanos más que a otros. Cerrar estas brechas de inequidad es una tarea impostergable del gobierno central pero también de los gobiernos locales conforme a sus competencias.

Después de varios años de implementación a partir del 2013 los resultados en algunas comunidades no han sido tan satisfactorios, existen avances en la comunidad parroquia Veintimilla, Ciudad Guaranda, Provincia Bolívar, todavía el problema del agua persiste, son afectadas las vías de acceso por las fuertes lluvias. Es conveniente prestar más apoyo a esa comunidad y aprovechar sus fortalezas como ocurrió en una comunidad en Cuenca que buscando resolver un problema de contaminación, descubrieron el potencial turístico de la zona, generando fuentes de empleo y cooperación en esa comunidad.

\section{Bibliografía.}

Alder, V. (30 de octubre de 2013). 8 temas que deben cuidar las ciudades para mejorar su calidad de vida. Obtenido de BID: Ciudades Sostenibles: https://blogs.iadb.org

Borjas, C. (Julio 2007). La asociatividad de las organizaciones sociales del sector subtrópico de la provincia bolívar. Revista Observatorio de la Economía Latinoamericana, Ecuador, http://www.eumed.net. Obtenido de http://www.eumed.net

Castro, F. (2013). La organización comunitaria defiende el desarrollo y ordenamiento territorial. Obtenido de Secretaría Técnica: Planifica Ecuador: http://www.planificacion.gob.ec

Coello, C. (12 de octubre de 2016). Quito presentará iniciativa 'Barrio Saludable' en Habitat III. Obtenido de Redacción Médica: https://www.redaccionmedica.ec

El Telégrafo. (14 de enero de 2013). Este ambicioso programa involucra a las instituciones Malaria, Gobierno Provincial Autónomo de El Oro, Universidad Técnica de Machala, Ministerio de Inclusión Económica y Social, Aguas y Servicios, Dirección de Educación, Municipio de Machala, Juntas Parr. Obtenido de El Telégrafo: https://www.eltelegrafo.com.ec

La Hora. (29 de mayo de 2013). Ciudadela Veintimilla padece falta de agua. Obtenido de La Hora: https://lahora.com.ec

Revista Científica Mundo de la Investigación y el Conocimiento. 3 (3). pp. 545-556 
Gestión del barrio saludable y la organización socio comunitaria en el sector Los Tanques, parroquia Veintimilla, Ciudad Guaranda, Provincia Bolívar, periodo 2013

Vol. 3, núm. 3., (2019)

Mariela del Pilar Bedoya Paucar; Laila Stefanía Lucio Escudero; Nancy Renee Cando Yaguar

Maisanche, F. (05 de octubre de 2018). Evaluación de daños en cuatro cantones de Bolívar continúa. Obtenido de El Comercio: www.elcomercio.com

Martí, C. (25 de agosto de 2008). Organización comunitaria: Líderes comunitarios. Obtenido de Madrid Blogs: https://www.madrimasd.org

Ministerio de Salud Pública. (diciembre de 2016). Municipios Saludables en el Ecuador. Obtenido de Manual para la certificación de municipios saludables: https://www.paho.org/ec

Moreta, M. (26 de abril de 2017). El sistema vial de Guaranda y de Sigchos afectado por el invierno en Cotopaxi y Bolívar. Obtenido de El Comercio.: https://www.elcomercio.com

Navas, M. (Julio de 2013). Evaluación de la gestión de la calidad de los servicios de salud ofertados por el Hospital San Sebástian del Cantón. Obtenido de Universidad Politécnica Salesiana, Cuenca: https://dspace.ups.edu.ec

Ruales, J. (21 de noviembre de 2017). Autoridades afinan proyecto Barrios Saludables. Obtenido de Redacción Médica: www.redaccionmedica.ec 